\title{
Interview
}

An interview with Pharmacogenomics for the breast cancer special focus issue

Alan Ashworth speaks to Sarah Miller, Assistant Commissioning Editor

Alan Ashworth joined the Institute of Cancer Research (ICR; London, UK) in 1986 as a postdoctoral scientist in the Section of Cell and Molecular Biology. He is now the Chief Executive of the ICR, a position he has held since January 2011. Prior to this, he was Director of the Breakthrough Breast Cancer Research Centre (London, UK) at the ICR, where he continues to lead the Gene Function teams' research efforts in breast cancer genetics, genomics and therapeutics. Together with Anthony Swerdlow (ICR), he cofounded and codirects the Breakthrough Generations Study, a large and comprehensive study aimed at unraveling the mysteries of breast cancer causation. In 1995, he played a key role in the team that discovered the BRCA2 risk gene. 10 years later, he was instrumental in discovering the potential of PARP inhibitors as synthetic lethal agents in BRCA-mutated cells. In 2008, Ashworth was elected as a Fellow of the Royal Society in recognition of his work in mammalian genetics and breast cancer susceptibility genes, and he continues to be passionate about developing personalized cancer treatments.

- Following on from your post-doc position in Cell \& Molecular Biology, why did you decide to specialize in breast cancer genetics \& molecular pathology?

I got into this by accident rather than by design. As with most of my career decisions, it was not planned - accidents and coincidences have led me into a new area. I had originally established my laboratory working on gene regulation in the $\mathrm{X}$ and $\mathrm{Y}$ chromosomes and $\mathrm{X}$ chromosome inactivation. It went pretty well - I published a couple of papers in Nature on X chromosome inactivation, and I was very interested in pursuing this area.

The change came in 1994 when Mike Stratton (Sanger Centre, Hinxton, UK), who was working in the Institute of Cancer Research (ICR; London, UK) at the time, found linkage between a region of chromosome 13 and a new breast cancer gene, and he asked me to help in identifying the gene. At the time, gene discovery was in its heyday; this was just at the start of large-scale sequencing and one still had to go out and search laboriously for these genes. I had developed a reputation as somebody who had expertise in this area, so I came in as a sort of 'hired gun' to help out. This gene chase was a terrifically interesting time in my career and life, and after a whirlwind effort we were able to identify the BRCA2 gene. At the time I did not realize this, but this discovery really changed my life and outlook. I think what influenced it was that I saw that the gene discovery had an almost immediate impact on people's lives - women at risk of breast cancer could be tested for the mutation. I then gradually started to turn my attention more to breast and other cancer genetics because I found it terribly interesting that not only could we do fundamental science, but there was also this applied element that could hopefully improve the outcome of patients. So I gradually reconfigured my laboratory to concentrate more on breast cancer, and when the opportunity arose in 1999 to become the Director of the Breakthrough Breast Cancer Research Centre, I grabbed it with both hands.

- You have been very involved in developing personalized therapies based on genomics. How did you get involved in developing PARP inhibitors for $B R C A$ mutationrelated cancers?

Again, this was a lucky break, although I think if you are going to exploit such accidents, you need to have the right mindset. Based on our and other people's understanding of what the defect was in BRCA2-mutant cells - the fact that $B R C A 2$ was involved in DNA repair and

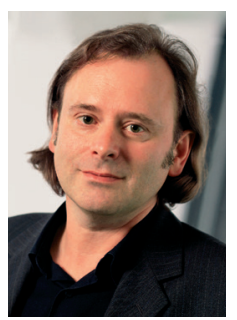

\section{Alan Ashworth}

The Breakthrough Breast Cancer Research Centre, 237 Fulham Road, London, UK alan.ashworth@icr.ac.uk 
the genome was unstable - we had already started thinking for several years that if we attacked that defect in the right way, we might actually kill cancer cells but not normal cells. The accidental part was meeting Steve Jackson, who founded a company called KuDOS (acquired by AstraZeneca in 2005). They had been developing PARP inhibitors as DNA repair inhibitors and also as chemosensitization agents. And so, talking and thinking together over a drink, we hatched this plan to test out these PARP inhibitors that he had developed on BRCA-mutant cells. Pretty much the next day some white powders arrived in the post. I had to persuade somebody in my laboratory to do the experiment because nobody was that keen at the time; this was a pretty speculative experiment.

We put the drugs on BRCA-mutant cells and we saw this absolutely spectacular response. We were able to kill these $B R C A$-mutant cells using a very, very low concentration of PARP inhibitors, and normal cells appeared to be hardly touched. It was such a brilliant result that it was too good to be true - we did not quite believe it at first, but pretty soon it just felt right. This was a genuine 'synthetic lethal' effect. Synthetic lethality is of course a genetic term originally coined in the 1940s, but not really exploited in cancer therapy at all. However, it really is a pure genetic concept that describes a particular interaction of two genes, and what we had done is hit the genetic interaction between PARP inhibition and $B R C A$ mutation, which together are lethal in cells. So we seemed to have hit on exactly the right combination.

What was also thrilling about this discovery was that the clinical trial started only a matter of months after the paper was published. The PARP inhibitors were already in clinical development, but essentially to give to unselected patients. What we are able to do is divert them away from all comers and say the best result might be in this small population of $B R C A$ mutant tumors. That meant we saw the first responses just a few months after publication of our paper describing our initial results. Again, that was an unbelievable moment. This really did seem too good to be true; that something in the laboratory could translate with such an effect in the clinic. Often, one gets results in the laboratory that do not translate when tested in the clinic for all sorts of reasons; however, here we saw these terrific responses. I have had the privilege of meeting a number of patients who have taken the drug and benefited from it, some of whom have had their lives extended by the treatment. Similar results were seen in Phase II. Although there are still some hurdles to overcome, as usual when you get to the late-stage clinical development, but I remain very confident that PARP inhibitors as a single agent will end up being licensed for the treatment of $B R C A$-related cancers, and beyond that, in other contexts that also have defects in that pathway.

\section{- Could you briefly describe the Breakthrough Breast Cancer} Research Centre \& the work in your laboratory? How do you hope this will inform future breast cancer therapies?

The Breakthrough Breast Cancer Research Centre gave me the opportunity to create a whole research center from scratch, so we ended up with 120 people working on everything from epidemiology and population genetics all the way through to targeted therapies, in a coordinated fashion. I do believe that if we are to make genuine progress in cancer, we do need to have integrated approaches with biologists, oncologists, pathologists, chemists, pharmacologists, mathematicians and others all working together. In this environment, my laboratory is trying to harness genetic dependencies to create new therapeutic approaches to cancer. We are integrating standard 'omics approaches with analysis of genetic dependencies on a very large scale. By doing this we hope to build on what we did with PARP inhibitors to find synthetic lethal interactions in multiple other cancer types. By having close clinical links we aim to bring benefit to patients from our discoveries as soon as possible.

What motivated you to design \& lead the Breakthrough Generations Study with Anthony Swerdlow?

This happened within the first couple of years of the Breakthrough Breast Cancer Research Centre. It was Tony who came to me with the idea of doing a large-scale cohort study for breast cancer, and my 
input was to think about the genetic aspects; the aim was to combine classical epidemiology with genetics. As a cohort study, it recruits women who by and large have not had breast cancer - a few have, but most have not - and then some, approximately one in nine, will develop breast cancer over time. That is the best design to tease out what the relationship is between genetics and lifestyle and environmental factors. So it is a very long-term program - up to 40 years of study. We wanted to create something that would build over time and was definitive.

What we found when we launched the study was that we had women queuing up to join, it was unbelievable actually. We had 10,000 signed up in the first couple of days. This tremendous response is a testament to the desire of people to contribute to scientific research and to help others. We now have questionnaire data and blood samples from over 100,000 women. There are other larger studies of breast cancer, but we believe ours is one of the most comprehensive in the sense that we have both very large amounts of lifestyle information and blood samples that allow us to analyze the DNA, hormones and plasma factors. We also have permission to gather all of the medical records over time from every woman taking part, and we can take further blood samples. So over time it will build into a huge resource.

We are already starting to use it to unpick some of the relationships between lifestyle, breast cancer risk and genetics. I think in the next 5 years it will really come into its own with the modern genetic techniques of genome sequencing and we are sure to see a huge volume of data. So I am very pleased that we started it, and in recruitment terms it has been a terrific success.

\section{How do you hope the findings from the studies, such as Breakthrough Generations, will impact on breast cancer prevention or treatment?}

I think we all agree that the cause of breast cancer is multifactorial; there is no cigarettes-and-smoking equivalent for breast cancer like there is in lung cancer. So to understand the causation of breast cancer, we need to understand what the genetic landscape is. We are getting close to that now with pure genetics studies; however, having the influence of lifestyle on top of that is, I think, the added benefit of the Breakthrough Generations Study. What I hope we can achieve, not just by this study, but by others as well, is an algorithm that can, for most women, give an accurate representation of what their risk of breast cancer is. It may well be that, for the majority of women, the risk of breast cancer is really quite low. However, if we can define the subset with enhanced risk, we can actively screen them; so rather than mammography screening everyone, we just screen that subset. Early detection is critical to improving breast cancer prevention and treatment. It also allows us to think about intervening in prevention studies - that is really the 'Holy Grail,' but it is fraught with difficulties. Most pharmaceutical companies are not really interested in prevention studies because by the time that you have proven your drug works in prevention, it is off patent and they will not make any money from it. So we are going to need to build an infrastructure to start to test out some of these hypotheses.

What do you see as being the opportunities in \& major obstacles to the implementation of personalized breast cancer medicine in the next 10 years?

What is already happening in a small number of centers, including our own, is a partial genome work-up on normal DNA and the tumor DNA, but this will quickly extend to the total genome structure. This will be used to map out the entire disease course of all cancers. So at presentation, it will be used to inform diagnosis and prognosis. Then it will be predictive to inform what treatment the patient should receive; for some it will indicate that no treatment is required apart from surgery because that would be sufficient to achieve a cure. For those unfortunate enough to relapse, we will take further biopsies of the tumor - again, a genome work-up to identify which clone has grown out. We could then modulate the treatment based on that and hopefully get a response in some, but again some will relapse. Taking biopsies will really allow us to study the natural history of the disease all the way through 
the disease course, putting genomics at the heart of cancer medicine.

There are significant challenges all the way through this, but for me I think the key challenge is heterogeneity. A tumor is not simply a clonal, homogenous mass, rather it contains lots of subclones that have genetic variations which may well make them resistant to drug treatment, and as a result, these clones may well grow out. So we need to find out what that clonal variation is early on. One has got to think of the Darwinian principles of speciation here; what you have got is a species of cancer that grows out because it survives when the others are killed. What we do not understand very well is how those principles operate in people being treated. Using very deep DNA sequencing we can start to map this out. I think that will give us much better knowledge of who might respond and when and how they might relapse. I think that will lead to better outcomes in cancer.

Another challenge is computational: we are starting to get a lot of data and we have to store all of this. There are also challenges surrounding interpretation: how do we make data available to healthcare professionals in a form that is useful to them? Clearly a genome sequence is of no immediate use - most of it is irrelevant - but we need to store that information until it becomes relevant. So I think there is a lot do in the terms of education and consideration of how we communicate genetic information so that oncologists in the clinic can use the information.

Even though there are immense challenges in integrating genomics in medicine, I am confident that it can be achieved and am very positive about the future.

\section{Financial \& competing interests disclosure}

The author may benefit financially from the development of PARP inhibitors through patents held jointly with AstraZeneca through the Institute of Cancer Research 'rewards to inventors' scheme. The author has no other relevant affliations or financial involvement with any organization or entity with a financial interest in or financial conflict with the subject matter or materials discussed in the manuscript apart from those disclosed.

No writing assistance was utilized in the production of this manuscript. 